\title{
Optimisation of post-disaster assisted self-build housing construction and labour safety in developing countries
}

\author{
Maria del Mar Casanovas-Rubio ${ }^{1, *}$, S.M. Amin Hosseini ${ }^{1}$, Albert de la Fuente ${ }^{1}$, Oriol Pons ${ }^{2}$ \\ ${ }^{1}$ Department of Civil and Environmental Engineering, Universitat Politècnica de Catalunya / Jordi \\ Girona 1-3, 08034 Barcelona, Spain \\ ${ }^{2}$ Department of Architectural Technology, Universitat Politècnica de Catalunya / Diagonal 649, 08028 \\ Barcelona, Spain
}

E-Mails: mar.casanovas@upc.edu (M. C.-R.); amin.hosseini@upc.edu (A. H.); albert.de.la.fuente@upc.edu (A. F.); oriol.pons@upc.edu (O.P.)

* Author to whom correspondence should be addressed; Tel.: +34-934-016-530

\begin{abstract}
Natural and man-made disasters and conflicts occur constantly around the world, leaving displaced and vulnerable people every year, especially in the developing countries. Among other urgent necessities, this population needs to rebuild their houses. Due to the scarcity of resources in these disaster contexts, many of them opt for self-build or community-based construction of simple houses. These labour methods can be a solution in these situations, maybe the only one available, but arise other problems such as a scarce awareness and prevention of the labour risks and limited construction knowledge and skills. Taking into account all the factors, a possible solution to this, proposed by the authors, could be an 'assisted self-build or community-based construction'. That is to say, a selfbuild or community based construction, in which the labour has been specifically trained in the occupational risk prevention and construction materials and techniques they are going to use. This involves a previous analysis with the following steps: (1) study of the local and traditional materials and construction techniques of vernacular houses; (2) study of how they could be improved by incorporating new current construction knowledge; (3) design of different low-tech but high-knowledge housing appropriate for self and community build; (4) evaluation of the sustainability of the alternative designs, including the labour risks during construction, and selection of the best one; and (5) definition of the teaching content and plan. The selected design should integrate the local and traditional construction materials and knowledge, which are usually available and sustainable, with the current knowledge, in order to improve the traditional design and make it more resistant to natural hazards such as earthquakes and, at the same time, create a more comfortable house. The
\end{abstract}


labour risks during self-construction should be evaluated, the construction process adapted in order to minimise the risks, and preventive measure defined.

Keywords: post-disaster housing construction; developing countries; labour methods; selfbuilt construction; community participation; labour risks; occupational risks; sustainability assessment; multi-criteria decision analysis.

\section{Introduction}

Natural disasters are due to a complex combination of natural hazards and disastrous human actions (Blaikie et al. 2014). On average, natural disaster affected 218 million people per year between 1994 and 2013 (CRED 2015). Displaced population (DP) who lost their home in the wake of natural disasters need somewhere to recover the normality of pre-disaster situation (Davis 1978). This need could be solved by means of temporary and permanent housing. Meanwhile, the short-term requirements should not be cause of forgetting the long-term requirements and those aspects associated to sustainability. In this sense, the disaster management needs to change the response and recovery strategy to sustainable hazard mitigation (Pearce 2003) (cited by Hayles 2010). Furthermore, Nakagawa and Shaw (2004) stated that natural disasters could be opportunities for sustainable reconstruction. Beyond the abovementioned facts, high-quality sustainable buildings is one of the human rights (Schneider 2012). These accommodations could be provided by different labour methods including: community, self-help, contracted, and direct labour methods.

Self-help method is used when the affected people have abilities to erect a shelter for themselves (DFID et al 2012). This fact normally happens in rural areas or small towns, where people have already had this experience. Although the abilities of the affected communities can help to erect temporary shelter and housing quickly, they could also create some problems, especially when this method is applied providing permanent housing. In other words, if previous building of affected community have been damaged/destroyed due to natural hazards, it means the previous building technology must not be applied again. In this regard, Caballero Moreno et al. (2018) stated that these buildings are usually vulnerable in terms of resisting to future natural hazards (Caballero Moreno et al. 2018). Thus, other more resistant technologies need to be applied, or at least the previous construction technologies of the affected area should be improved to have robust buildings against future hazards based on the engineering codes and standards.

In this regard, affected people, who know how to construct the previous buildings, are involved in construction activities of new buildings, which need higher expertise than DP's experiences. Therefore, the lack of enough building construction knowledge by DP together with inappropriate interference of the affected people in buildings' technical details, have negative impacts on the quality of these buildings. Additionally, the high-technology buildings need more technical details and components than the previous buildings, which normally implies the use of different construction technologies and processes. This needs to be considered together with professional management, such as standard components, occupational risks, and so on. However, the affected people's approaches are usually far from these standards. Therefore, management skills should be applied to achieve success when there are two issues: (1) poor security, and (2) complex works (Collins et al. 2010). Although 
considerable guidelines exist to assist the affected people on how to build their shelters, there are no/little research studies about the occupational risks of post-disaster housing construction.

Consequently, the objectives of the research presented in this paper are threefold: (1) to provide an overview of the four different labour methods in post-disaster housing; (2) to propose a method to evaluate the occupational risks; and (3) to define a value tree that enables the sustainability assessment of the different post-disaster housing construction technologies based on the multi-attribute utility theory.

\section{Labour methods of post-disaster accommodations}

\subsection{Top-down and bottom-up approaches}

According to Dikmen et al. (2012), post-disaster housing recovery programs can be organized into top-down and bottom-up approaches in terms of the beneficiaries' roles and organizational forms. The top-down approach is based on governmental decisions and actions without giving a role to DP. This approach is highlighted by standardization and technology-oriented solutions in order to shorten delivery time and minimize public expenditures. However, this approach does not provide other requirements, such as cultural and local conditions (Johnson 2007a). In contrast, the bottom-up approach considers all beneficiaries' requirements by empowering DP (Dikmen et al. 2012). This approach has proven to be more successful in terms of adaptation to culture, local skills, and climate conditions (Johnson 2007a). Many factors have to be considered in order to classify a housing recovery program into the top-down or bottom-up approach.

\subsection{Definition and analysis of the labour methods}

Disaster management decision-makers need to specify the labour methods based on each postdisaster reconstruction scenario. According to Collins et al. (2010), in order to determine labour types of post-disaster accommodation, it is required to consider the four factors: (1) availability of local skilled and unskilled labour, (2) organizational and local requirements, (3) tools and equipment, and (4) transportation conditions (Collins et al. 2010). The DFID et al. (2012) considers four labour methods: direct, contract, community, and self-help, which are explained next.

\subsubsection{The direct labour method}

Direct labour is a method when aid agencies and organizations hire and manage labour directly. In this case, organizations should be experienced and have their own experts, such as architects, civil engineers, and so on. In this method, the organizations should be familiar with local culture and potentials.

\subsubsection{The contract labour method}

The contract labour method takes into account construction approaches to provide DP's accommodations by hiring a contractor. According to Tas et al. (2007), the contract approach is more frequently used compared to the others in order to provide permanent housing. Although, this method does not consider any role for DP, it is highly significant for the construction delivery time and quality. 
Additionally, sometimes, the contract labour method is the only one that can be used in some areas due to DP features, such as lack of skills, free time, willingness to participate, etc.

\subsubsection{The community-participation method}

The community-participation method embraces construction approaches when DP together with or without the host community are willing to provide the post-disaster accommodations voluntarily. This method has been frequently used for permanent housing construction as well. It is applied on the basis of local construction methods for one or two-storey buildings (Caballero Moreno et al. 2018). The community participation in the construction process can go beyond solely being a labour force, and further play a role at other phases (Davidson et al. 2007). Furthermore, Davidson (2009) stated that even for building construction in normal situations, in order to achieve appropriate organizational forms, it is necessary to consider stakeholders' characteristics, such as culture. Additionally, it should be emphasized that the organizational strategy has great impact on the supervisors' roles, which is one of the key issues for post-disaster accommodation provision (Gharaati and Davidson 2008).

In general, the participation method, which has been planned by experts, and has been admired by several researchers, also has some problems that should be considered. Sometimes DP are not interested in community participation strategy based on diverse reasons. In this regard, Sliwinsky (2007) found that the community-participation approach to temporary housing (TH) provision could fail depending on the DP's motivations and characteristics. In this situation, the other strategies should be used. For instance, aftermath of earthquake and Tsunami in Aceh, 2004, the DP did not desire to corporate housing construction, and consequently, some agencies forced to employ contractors for constructing the buildings (Da Silva 2010). Additionally, the number of DP considerably affects the decision-making process and choice of a final strategy.

\subsubsection{The self-help method}

According to Collins et al. (2010), self-help labour is a construction method in which families are doing the construction activities under management of aid agencies. Self-help labour method is applied based on one of the two possible situations: unplanned or planned. DP provides unofficial TH as a selfbuilt accommodation when decision-makers have not provided a comprehensive TH plan or are slow in providing it. However, sometimes, agencies and organizations plan to apply self-help labour strategy. An example of DP that provided shelters for themselves as TH is the Colombian recovery program after the Armenia earthquake, 1999 (Johnson et al. 2006).

Doberstein and Stager (2013) mentioned that vulnerability of informal housing could be due to several factors, such as poor housing, location, design, etc., based on probable hazards. This fact is established for self-help method as well. It is vital to consider possible hazards in order to define the most suitable building technology in each case while considering other factors. In this regard, Caballero Moreno et al. (2018) defined the main requirements of self-help method: (1) light construction elements, (2) based on minimum building knowledge, (3) without complex machinery, (4) raw material that could easily be accessible, and (5) cultural acceptability.

Self-help labour method can conceptually be organized into the bottom-up approach. However, sometimes, agencies or, in general, decision-makers impose some restrictions to DP. Therefore, in those cases, it is difficult to categorize the self-help labour as a bottom-up approach. For instance, 
decision makers force DP to follow a specific building plan because, if each building has a different design plan, it is difficult to control the construction quality. In return, decision-makers provide them with construction materials or funds.

The other issue of these last two methods (community and self-help) that should be considered is the special features of the DP. In this regard, according to Blaikie et al. (2014), some people are more vulnerable in the context of various hazards due to their income level, occupation, gender, disabilities, health status, caste, age, immigration status, etc. Moreover, the main priorities (concerns) of DP could be something different to housing provision. For example, if DP should take care of a person from their family due to the aforementioned vulnerabilities, it is difficult for them to participate in housing reconstruction.

\subsection{Conclusions}

Therefore, based on the mentioned features of the methods, decision-makers need to assess the integration of local characteristics and housing features to determine suitable construction approaches. According to Apoport (1969), building typologies have been influenced by climate conditions and culture. Therefore, it is necessary to consider local characteristics.

Decision-makers face a dilemma between applying the strategies used before the disaster, which could not successfully resist the natural hazards, or new buildings strategies, which are not well known by the DP. If the previous strategies are selected, all their weaknesses and disadvantages need to be carefully considered and modified in order to reach the build back better concept. For instance, the core house was used as a temporary and permanent housing project after the central Java earthquake, and it has also been used as a low-cost housing program in Indonesia for decades (Kondo and Maly 2012).

\section{Labour Risk Assessment of post disaster-housing construction}

Labour risk prevention is a fundamental social factor that should also be considered when selecting the labour method and construction technology in post-disaster housing construction. However, little research has been found on the labour risk of post-disaster construction. The research presented herein proposes a method for assessing occupational risks in construction and a first approach to specifically adapt it to post-disaster housing construction in developing countries.

\subsection{Occupational Risk Index}

The Occupational Risk Index defined in Casanovas et al. (2014) enables the assessment of the occupational risks in construction according to the activities performed, as shown in Equation (1).

$$
O R I=\sum_{i} O R I_{i}=\sum_{i} \frac{P_{i} \times C_{i}}{1000} \times E_{i}
$$

Where $i$ is a risk associated with an activity, $O R I_{i}$ is the Occupational Risk Index of risk $i, P_{i}$ is the probability of occurrence of an accident given risk $i, C_{i}$ is the severity of its most probable consequence, and $E_{i}$ is the exposure of the workers to risk $i$ expressed in units of time (hours).

A list of risks that may be present in construction and related construction activities are provided in Casanovas et al. (2014). Furthermore, each risk-activity was assigned a rating of probability $\left(P_{i}\right)$ and severity of the consequence $\left(C_{i}\right)$ by using the ratings in Tables 1 and 2 respectively. For example, the 
risk of falls to lower levels from outside openings in façades was considered an unusual sequence $(\mathrm{P}=3)$ with the consequence of extremely serious injury or fatality $(\mathrm{C}=20)$. The exposure time needs to be defined according to the person-hours dedicated to construction activities close to openings in façades according to the specific construction project and plan.

Table 1. Ratings for the probability of an accident (adapted from Fine 1971)

\begin{tabular}{|l|c|}
\hline Classification & Rating \\
\hline Most likely and expected result should the hazard event occur & 10 \\
\hline Quite possible, not unusual & 6 \\
\hline Unusual sequence & 3 \\
\hline Remotely possible & 1 \\
\hline Has never happened but it is conceivable & 0.5 \\
\hline
\end{tabular}

Table 2. Ratings for the severity of the most probable consequence of a potential accident (adapted from Fine 1971)

\begin{tabular}{|l|c|}
\hline Classification & Rating \\
\hline Catastrophe, numerous fatalities & 100 \\
\hline Multiple fatalities & 50 \\
\hline Fatality & 25 \\
\hline Extremely serious injury (amputation, permanent disability) & 15 \\
\hline Non-serious disabling injury & 5 \\
\hline Minor injury (minor cuts, bruises and bumps) & 1 \\
\hline
\end{tabular}

\subsection{Occupational risk assessment in developing countries: application to Iran}

As explained in Casanovas et al. (2014), the ratings of probability and consequences depend on the technological development of the region and construction company as well as the approach taken to preventive measures. The ratings provided there correspond to the construction companies in Spain and should be used as a guide that may not apply in all cases. For other levels of technological development and safety management practices, these probabilities and severities of the consequences should be accordingly adjusted by the local construction health and safety experts. For lower levels of preventive measures such as in some developing countries, the probability and consequence ratings and, therefore, occupational risks, could be higher.

In this regard, a preliminary qualitative research has been carried out in a country classified as a developing economy by the United Nations such as Iran (UN, 2014). A first round of interviews have been carried out with managers of construction companies in Iran. The preliminary qualitative results suggest that the Iranian construction managers perceive the following risks and activities as the highest ones in building construction in Iran:

- Falls to lower levels from different places and while doing different activities: tower cranes, scaffoldings, hollow spaces, shafts, cut and fill batters, works on decks, conventional formworks, installation and execution of steel structures (light and heavy), installation of 
reinforcing bars, concrete-pouring from high levels, brickwork from high levels (indoor and outdoor), stonework from high levels (indoor and outdoor), plasterwork and staining from high levels, manual demolitions, and using temporary stairs.

- Impact injury from falling objects and projectiles in demolitions and rubble removal, falls of tools and construction materials due to human error or wind blow.

- Direct or indirect electrical contact due to works in proximity to power lines, and works with electrical equipment and electrical equipment under wet conditions.

- Burns caused by: fire in works close to fuel pipelines, welding, drilling and cutting, welding in proximity of flammable materials along the wind direction, accidental burn of flammable material in the storehouse, accidental explosion of the bottle of gas used for welding, burning of the waste generated in the construction site, accidental burn of standard and self-built heating devices, self-built non-standard wirings, smoking, and cooking.

- Entrapment and subsequent suffocation due to a landslide in earthmoving, shaft excavation, collapse of the retaining wall or contiguous building collapse.

- Suffocation or poisoning in confined spaces: works in lower floors and heating system with natural gas.

- Cuts, blunt trauma, and other injuries due to light equipment

- Acute dust and toxin poisoning in general demolitions, demolitions of hospitals, factories, and other places that may contain toxic substances, works in proximity to cement silos.

- Collision with or entrapment by a moving load due to its movement or detachment in mechanical load handling: cranes and self-propelled industrial trucks, winches and other means of mechanical load handling.

- Collision with or running over by heavy equipment or heavy-goods vehicle.

- Other activities that may cause accidents are: fights between workers, physical pranks, talking with mobiles, etc.

The probability of occurrence of these accidents and the severity of its most probable consequence of these and other possible risks in construction should be evaluated by health and safety experts in developing countries. Being aware of the construction risks, will allow the decision makers to take better decisions when it comes to chose the labour method and construction technology to be used in post-disaster building construction. It will also enable the adoption of adequate preventive measures and, finally the reduction of accidents.

\section{Sustainability assessment tool for post-disaster permanent housing}

As explained in section 2, there are several labour methods that can be applied in post-disaster permanent housing reconstruction. There are also several construction technologies and materials that can be used to that end. Hosseini et al. (2016) analysed some of them for temporary housing units. In order to chose the best construction alternative, many factors/criteria of different nature should be considered such as cost, occupational risks, comfort for the future dwellers, etc. Most of the times, the best alternative regarding one criterion is not the best alternative regarding other criteria. For example, the best alternative regarding the dweller's comfort in terms of thermal and acoustic isolation may not be the cheapest alternative. Therefore, the best solution would be a compromise solution between all the factors, which is the closest solution to an ideal solution between all the feasible alternatives. 
In these cases, multi-criteria decision analysis (MCDA) is a valuable tool to assist decision makers in the decision making process. MCDA an, specifically, the multi-attribute utility theory (MAUT) (Keeney and Raiffa 1976) can help decision makers to organise their ideas into criteria and subcriteria and quantify their preferences in order to make decisions in a more objective and systematised way. MAUT has already been successfully applied to decision making in construction and to evaluate sustainability in construction.

Based on this theory, a value tree for evaluating the housing alternatives of post-disaster permanent housing was defined (Figure 1). It is the result of interviews with Iranian and Spanish experts in postdisaster management and reconstruction, and decision making and the consideration of some previous related publications (Hosseini et al. 2016, Casanovas-Rubio and Ramos 2017, and Casanovas-Rubio 2014). The value tree consists of three hierarchy levels that are the requirements, the criteria and the indicators. The criteria and indicators defined should be discriminative between alternatives. The alternatives to be compared are the different construction technologies and materials and labour methods.

Figure 1. Value tree for decision making on post-disaster permanent housing.

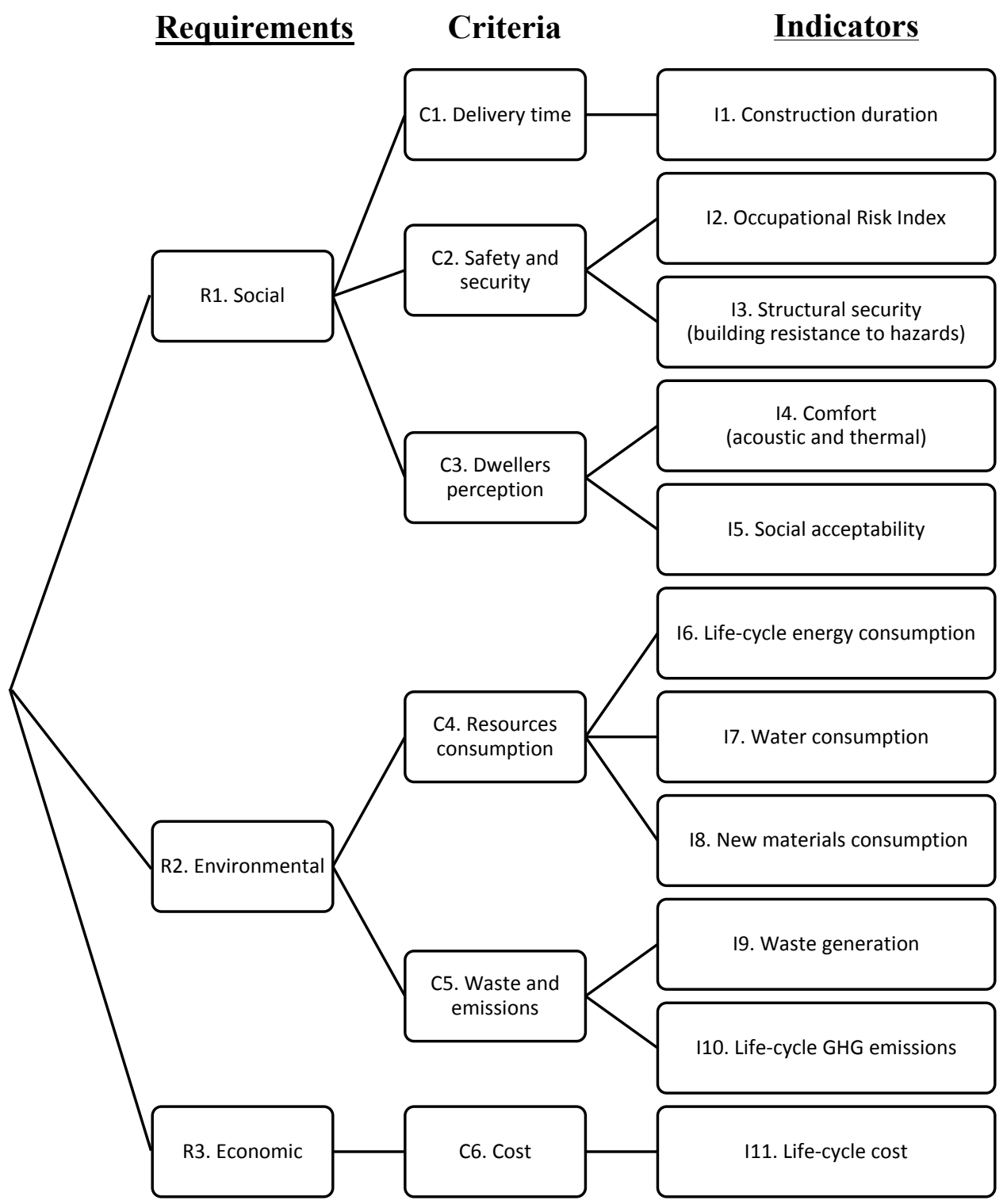


The value tree includes the three pillars of sustainability: social, environmental and economic. The social requirement (R1) takes into account how the different constructing technologies affect the construction workers and the future dwellers of the permanent post-disaster housing. The environmental requirement (R2) considers the environmental effects due to the inputs and outputs of the construction technologies. Finally, the economic requirement (R3) assesses the necessary expenditures for each alternative during its entire life-cycle.

Within the social requirement (R1), three criteria were found to be relevant:

- The delivery time $(\mathrm{C} 1)$ of the post-disaster permanent housing in order to limit the time that the affected people live in TH. It is measured by a single indicator which is the construction duration (I1) in time units.

- The safety and security (C2) of the construction labour as well as the future dwellers. It is measured by two indicators. The first indicator is the Occupational Risk Index (I2), which measures the occupational risks during construction due to the activities performed. This indicator was addressed in section 3. The second indicator is the structural security (I3) which considers the building resistance to earthquakes, fires, flooding and, in general, to the hazards that are locally relevant.

- The dwellers perception (C3) considers two indicators: the acoustic and thermal comfort (I4) provided by the housing and its social acceptability (I5) due to cultural, socio-economic and aesthetical reasons.

Within the environmental requirement (R2), two criteria were found to be relevant:

- The resource consumption (C4), which includes three indicators. The first indicator is the lifecycle energy consumption (I6) which includes the energy consumption in the manufacturing and transportation of the construction materials, as well as in the operational and demolition stages. The second indicator is the water consumption (I7) during the manufacturing of construction materials, construction, and demolition stages. The operational stage is not considered in the analysis because it does not discriminate between construction technologies (alternatives). The last indicator is the new material consumption (I8) in the construction stage. The reused or recycled materials are not added to the consumption as a way to reward these practices.

- The waste and emissions (C5) generation, which, in turn, contains two indicators. The waste generation (I9) assesses the amount of waste generated during the construction and demolition stages that is not going to be reused or recycled. The life-cycle GHG emissions (I10) indicator assesses the greenhouse gas (GHG) emissions in the cradle-to-grave or cradle-to-cradle stages of the buildings.

The economic requirement (R3) considers a single criterion, cost (C6), which is evaluated by means of a single indicator, life-cycle cost (I11).

\section{Discussion}

According to the literature review as well as the experiences of the authors, in order to determine the most suitable construction technology and labour method, which are intertwined with each other, it is necessary first to determine the feasible set of alternatives to the particular case study. This implies 
to firstly consider a combination of three aspects. These are: possible risks, affected community characteristics and capacity, and construction technologies, as shown in Figure 2.

In order to consider risks, it is required to study previous risks based on previous natural hazards with different intensities as well as new possible hazards. The socio-economic and socio-cultural characteristics and capacities of the affected community need to be assessed, especially the local capacity after the event. Additionally, possible construction technologies, even those that have not already been used in the affected area, should be assessed. It should be emphasized that not-used technologies can be applied provided that their requirements, such as skilled supervisors, building materials and resources, and cultural acceptance by DP are met. It should be mentioned that construction technologies have been considered as a separated aspect and not a part of the local capacity. Furthermore, besides local resources for the construction activities, receiving resources from outside of the affected area needs to be considered.

Figure 2. Aspects to determine the feasible labour methods and construction technologies (feasible alternatives) for post-disaster housing construction

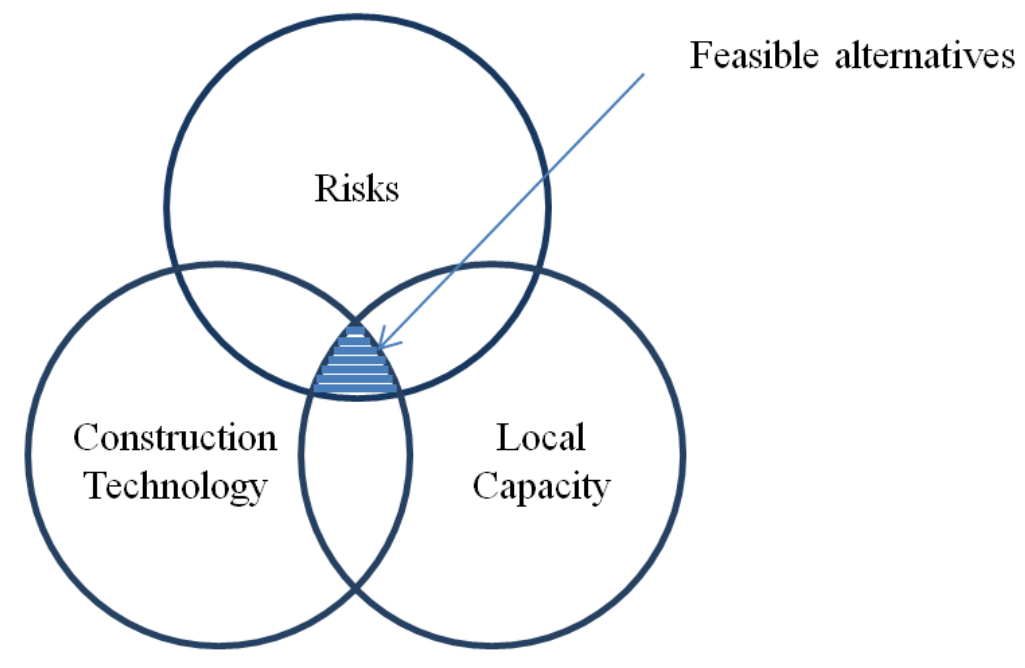

Once the set of feasible alternatives has been determined, the alternatives should be assessed and ranked by means of a MAUT tool and the best alternative should be selected. To that end, a value tree with requirements, criteria and indicators have been provided in section 4 . In a next step, the relative importance of the requirements, criteria, and indicators should be assessed by the different stakeholders of post-disaster reconstruction.

\section{Conclusions}

This study provides a general view about labour methods of post-disaster accommodation by considering its advantages and disadvantages. It also proposes a method for evaluating the labour risks in construction of post-disaster housing and presents a preliminary analysis of the labour risks in construction in Iran. Additionally, this research presents a decision making model based on the MAUT that enables the assessment of the sustainability of the different technologies and labour alternatives for post-disaster housing construction. A value tree with requirements, criteria and indicators was defined. 
The conclusions on post-disaster housing construction that can be draw from this analysis are:

- There are four main labour methods for post-disaster housing construction: direct, contract, community, and self-help.

- Self-help and community participation labour methods are not always the most suitable approach for dealing with post-disaster accommodation provision.

- Sometimes the conceptual methods are different from what is done in practice. Consequently, it is necessary to find a right way to pass from conceptual definitions to suitable actions.

- Labour risk prevention is a fundamental social factor that should also be considered when selecting the labour method and construction technology. However, little research has been found on this topic. The Occupational Risk Index could be used for assessing the occupational risks in post-disaster housing construction. The probability and consequence ratings could be easily adjusted to the study case if considered necessary.

- Considering the complexity of the decision due to the multiple factors and stakeholders that should be taken into account, MAUT can help to assess and select the best and most sustainable construction technology and labour method to be used in post-disaster housing construction. In this regard, a value tree with requirements, criteria and indicators has been proposed in this research.

Further work is required in order to adjust the probability and consequence ratings of occupational risks in construction for regions with different levels of technological development and risk prevention management such as developing countries by means of interviews with local health and safety experts. Further work is required to assess the relative importance of the requirements, criteria and indicators of the value tree by means of interviews to the relevant stakeholders in post-disaster housing construction.

\section{Acknowledgments}

The authors gratefully acknowledge the financial support received from the Universitat Politècnica de Catalunya's Centre for Development Cooperation under the 2018-U012 and 2017-U006 projects. The authors would further like to express their gratitude to the Islamic Azad University of Tehran, Kermanshah municipality, and the Housing Foundation of I.R. Iran for their collaboration. Finally, the authors would like to thank the interviewed Iranian construction experts.

\section{Conflict of Interest}

The authors declare no conflict of interest

\section{References and Notes}

Apoport, Amos. 1969. House form and culture. Prentice-Hall.

Blaikie, Piers, Terry Cannon, Ian Davis, and Ben Wisner. 2014. At risk: natural hazards, people's vulnerability and disasters. Routledge.

Caballero Moreno, William G., Inés Alegre, Jaume Armengou-Orús, and Antonio Aguado. 2018. "Self-construction in informal settlements: a multiple-criteria decision-making method for assessing sustainability of floor slabs in Bucaramanga, Colombia." Journal of Housing and the Built Environment: 1-23. doi:10.1007/s10901-018-9606-5. 
Casanovas, Maria del Mar, Jaume Armengou, and Gonzalo Ramos. 2014. "Occupational Risk Index for assessment of risk in construction work by activity." Journal of Construction Engineering and Management 140(1): 04013035-1- 04013035-9. DOI: 10.1061/(ASCE)CO.1943-7862.0000785

Casanovas-Rubio, Maria del Mar. 2014. "Metodología para la evaluación y seguimiento de procedimientos constructivos de forma sostenible e integrada." Doctoral Thesis Supervised by Gonzalo Ramos. Universitat Politècnica de Catalunya, Barcelona.

Casanovas-Rubio, Maria del Mar, and Gonzalo Ramos. 2017. "Decision-making tool for the assessment and selection of construction processes based on environmental criteria: Application to precast and cast-in-situ alternatives." Resources, Conservation \& Recycling 126: 107-117. DOI: 10.1016/j.resconrec.2017.07.035

Collins, Sam, Tom Corsellis, and Antonella Vitale. 2010. Transitional shelter: understanding shelter from the emergency through reconstruction and beyond. ALNAP. Accessed 10 10, 2014. www.alnap.org/pool/files/innovationcasestudyno5-shelter.pdf.

CRED. 2015. "The Human cost of Natural Disasters." The Centre for Research on the Epidemiology of Disasters.

Da Silva, Jo. 2010. Key Considerations in Post-Disaster Reconstruction .

Davidson, C. 2009. "The challenge of organizational design for manufactured construction." Construction Innovation 9 (1): 42-57. doi:10.1108/14714170910931534.

Davidson, C.H., Cassidy Johnson, Gonzalo Lizarralde, Nese Dikmen, and Alicia Sliwinski. 2007. "Truths and myths about community participation in post-disaster housing projects." Habitat International 31 (1): 100-115. doi:10.1016/j.habitatint.2006.08.003.

Davis, Ian. 1978. Shelter after disaster. Oxford: Oxford Polytechnic Press.

DFID, UKAID, IOM, Sida, and Shelter Centre. 2012. Transitional Shelter guideline. Geneva, Switzerland: Shelter Centre. www.sheltercentre.org/library.

Dikmen, Nese, Soofia Tahira Elias-Ozkan, and Colin Davidson. 2012. "Comparison of post-disaster housing procurement methods in rural areas of turkey." Open House International 37 (1): 28-39.

Doberstein, Brent, and Heather Stager. 2013. "Towards guidelines for post-disaster vulnerability reduction in informal settlements." Disasters 37 (1): 28-47.

Fine, William T. 1971. "Mathematical evaluation for controlling hazards." Journal of Safety Research, 3(4): 157-166.

Gharaati, Mehran, and Colin Davidson. 2008. "Who Knows Best? An Overview of Reconstruction after the Earthquake in Bam, Iran." Proceedings ofthe.

Hayles, Carolyn S. 2010. "An examination of decision making in post disaster housing reconstruction." International Journal of Disaster Resilience in the Built Environment 1 (1): 103-122. doi:10.1108/17595901111149141.

Hosseini, S.M. Amin, Albert de la Fuente, and Oriol Pons. 2016. "Multi-criteria decision-making method for assessing the sustainability of post-disaster temporary housing units technologies: A case study in Bam, 2003." Sustainable Cities and Society 20: 38-51. DOI: 10.1016/j.scs.2015.09.012

Johnson, Cassidy. 2007a. "Impacts of prefabricated temporary housing after disasters: 1999 earthquakes in Turkey." Habitat International $31 \quad$ (1): 36-52. doi:10.1016/j.habitatint.2006.03.002. 
Johnson, Cassidy, Gonzalo Lizarralde, and Colin H. Davidson. 2006. "A systems view of temporary housing projects in post-disaster reconstruction." Construction Management and Economics 24 (4): 367-378. doi:10.1080/01446190600567977.

Keeney, Ralph L., and Howard Raiffa. 1976. Decisions with multiple objectives: preferences and value tradeoffs. New York: John Wiley \& Sons.

Kondo, Tamiyo, and Elizabeth Maly. 2012. "Housing recovery by type of resident involvement-providing housing vs. mobilizing residents." The first international conference for International Society of Habitat Engineering and Design (ISHED). Shanghai. Accessed 7 18, 2014. http://www.tamiyokondo-lab.jp/pdf/essay/essay_29.pdf.

Nakagawa, Yuko, and Rajib Shaw. 2004. "Social capital: A missing link to disaster recovery." International Journal of Mass Emergencies and Disasters 22 (1): 5-34.

Pearce, Laurie. 2003. "Disaster management and community planning, and public participations: how to achieve sustainable hazard mitigation." Natural hazards 28 (2-3): 211-228. doi:10.1023/A:1022917721797.

Schneider, Claudia. 2012. Sustainable Reconstruction in Disaster-Affected Countries: Practical Guidelines. SKAT \& UNEP.

Sliwinsky, Alicia. 2007. "Social dynamics in participatory reconstruction: an anthropological analysis from El Salvador." 225-234.

Tas, Nilufer, Nilay Cosgun, and Murat Tas. 2007. "A qualitative evaluation of the after earthquake permanent housings in Turkey in terms of user satisfaction-Kocaeli, Gundogdu Permanent Housing model." Building and Environment 42: 3418-3431. doi:10.1016/j.buildenv.2006.09.002.

UN. 2014. World economic situation and prospects 2014. New York: United Nations. Retrieved from: https://unctad.org/en/PublicationsLibrary/wesp2014_en.pdf

(C) 2018 by the authors; licensee MDPI and IFoU, This article is an open access article distributed under the terms and conditions of the Creative Commons Attribution license. 